\title{
ANALOG TUNABLE GRATINGS WITH NANOMETER RESOLUTION
}

\author{
Chee Wei Wong, Wei-Chuan Shih, Yong Bae Jeon, Salil Desai, \\ Dennis Freeman, Sang-Gook Kim, and George Barbastathis \\ Massachusetts Institute of Technology, Cambridge, MA 02139
}

\begin{abstract}
We have designed analog tunable diffractive gratings with period tunable to within fraction of a nanometer and diffracted angular changes on the order of $1 \mu$ radian. We deform the gratings in both electrostatic and piezoelectric actuator designs, and demonstrate period changes of order $2 \mathrm{~nm}$, with limitation from measurement noise. Fabrication processes and results are presented. Actuator characterization and experimental measurements for both devices suggest applications in optical telecommunications and microspectroscopy.
\end{abstract}

\section{INTRODUCTION}

We here present a tunable MEMS grating which permits analog control over the diffraction angle, accomplished by analog deformation of the grating structure in the lateral direction. Prior work on digitally tunable gratings involves individual control of each grating beam or a set of beams (such as in the Grating Light Valve [1] and Polychromator [2]). Work on analog tunable gratings involves either thermal actuation $[3,4]$, or vertical and lateral movement of two vertically stacked grating structures [4]. Compared to these tunable grating implementations, our device concept trades deflection range for angular resolution. Applications for high-resolution analog tunable gratings include microspectrometers, external cavity tunable lasers, and thermal compensators for wavelength multiplexer-demultiplexers. In this paper, we describe two implementations: electrostatic and piezoelectric. The piezoelectric design is better suited for applications requiring smaller tuning range and ultra-fine tunability of below $1 \mathrm{~nm}$. The electrostatic design, on the other hand, is appropriate for broader range and coarser tunability.

\section{CONCEPTS AND DESIGN}

Analog tunability of our grating devices is achieved by lateral actuation forces on the grating structure, either by electrostatic comb-drives or thin-film piezoelectric actuators, as illustrated in Figure 1. By contrast, for the digital design, the tuning is achieved by pulling some of the grating beams downwards by electrodes beneath the grating. Intensity modulation is realized by controlling the beam height. The tuning resolution is limited to the beam width (approximately $1 \mu \mathrm{m}$ ). Theoretically our devices, given the lateral actuated deformation, can have resolution three orders of magnitude better than the digital versions. In the electrostatic device, the grating is comprised of suspended beams supported by flexures. In the piezoelectric device, the grating is deposited onto a thin sub-micron membrane, from which the membrane is deformed to create the desired grating period change.

Figure $2 \mathrm{a}$ illustrates the design schematic of the electrostatic device. Two comb-drive actuators [5] deform the periodic structure. The structure is composed of grating bars in the center window and flexures which connect each bar. The flexures determine the stiffness of the entire structure. The suspended structure is attached to the silicon substrate through four anchors.

The stiffness of the flexure can be estimated by a general formula $\mathrm{k}=\mathrm{Ew}^{3} \mathrm{t} / \mathrm{L}^{3}$, where $\mathrm{k}$ is the effective spring constant for one period, $\mathrm{E}$ the Young's modulus of the material, $t$ the thickness of the structure, w the width of the flexure beam, and L the length of

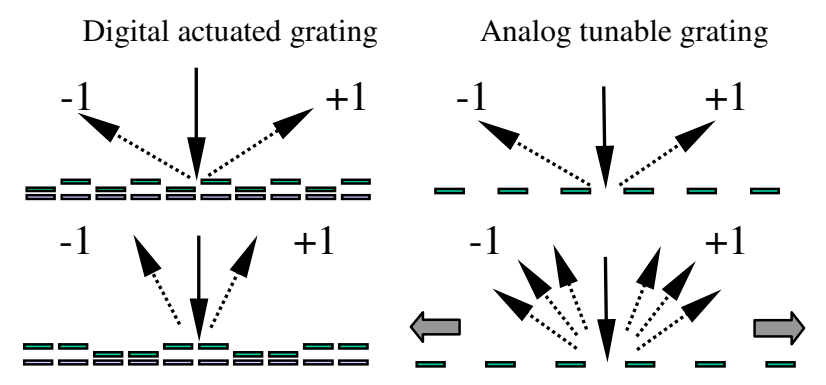

Figure 1. Actuation concept of an analog tunable grating, permitting analog control of diffraction angle.

the folded beam. The flexure stiffness is selected based on a tradeoff: low tuning voltage $(<30 \mathrm{~V})$ requires the device to be compliant, while the device should be sufficiently stiff so as to avoid modal resonance. Additional, the comb-drive actuators draw essentially no current; therefore, it operates with minimal power requirements. While the comb-drives deliver small force, usually limited to $\mu \mathrm{N}$ or less, the structure could be designed to be adequately complaint. The force can be estimated as $\mathrm{F}=-\mathrm{NetV}^{2} / 2 \mathrm{~g}$, where $N$ is the number of finger pairs, $\varepsilon$ is the permittivity of air, $t$ is the thickness of the structure, $g$ is the gap distance between two adjacent fingers, and $\mathrm{V}$ is the applied voltage. The minimum grating period is set by the resolution of the available lithography tool. Since the flexures on the sides of the grating must be defined, we find the minimum grating pitch is, at best, 4 times the design rule for $75 \%$ duty cycle or 6 times the design rule for $50 \%$ duty cycle.

(a)

(b)
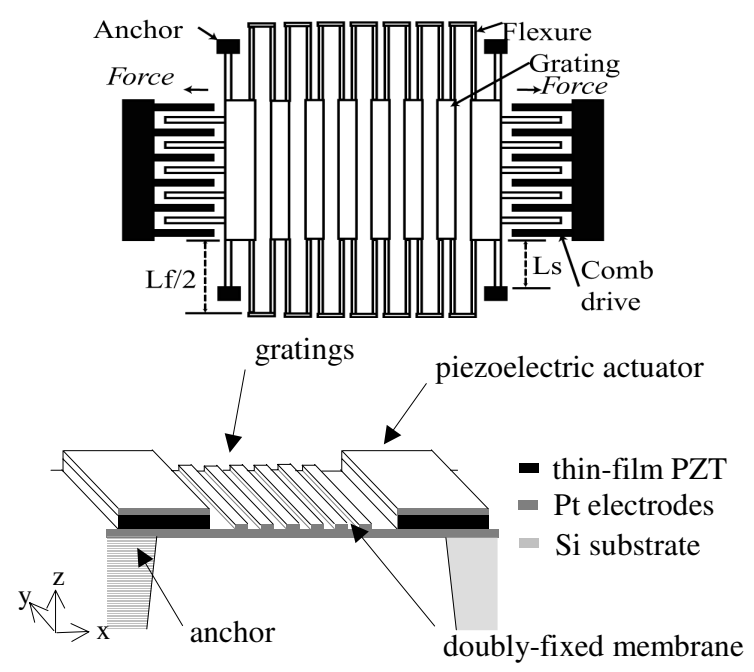

Figure 2. Design schematic: (a) Electrostatic comb-drive actuation, (b) Piezoelectric thin-film actuation.

For the piezoelectric device, the driving force is provided via the deposited thin-film lead zirconate titanate (PZT) actuators. Application of an electric field across the PZT film effects shrinkage of the film in the lateral direction, due to reorientation of the crystallographic domains. The membrane is correspondingly strained. The period of the diffraction grating, which is deposited onto the membrane, is thus progressively tuned and a linear response to the applied voltage due to the small strain range of less

\section{Travel support has been generously provided by the Transducers Research Foundation and by the DARPA MEMS and DARPA BioFlips programs.}


than $1 \%$. The doubly-hinged membrane design configuration, shown in Figure 2b, minimizes out-of-plane displacements of the gratings from either residual stress or the actuated piezoelectric films.

The in-plane $\mathrm{x}$-deflection $\delta_{\mathrm{x}}$ prediction, along the grating period axis, begins with a piezoelectric bimorph model [6] as

$$
\delta_{\mathrm{x}}=\frac{\mathrm{d}_{31} \mathrm{E}_{\mathrm{pzt}} \mathrm{A} \mathrm{V_{ \textrm {a } }}}{\mathrm{t}_{\mathrm{pzt}} \mathrm{k}_{\mathrm{x}}},
$$

where $k_{x}$ is the effective axial stiffness $=\sum_{i} E_{i} A_{i} / L, d_{31}$ the piezoelectric coupling coefficient, $\mathrm{E}_{\mathrm{i}}$ the material Young modulus, $\mathrm{A}_{\mathrm{i}}$ the cross-sectional area, $\mathrm{V}_{\mathrm{a}}$ the applied voltage, $\mathrm{t}_{\mathrm{pzt}}$ thickness of PZT layer, and $\mathrm{L}$ the $\mathrm{x}$-dimension beam length. Boundary conditions are then applied, with symmetry across the center of the membrane, and the solution reached iteratively. We note for an isotropic wide membrane, $\mathrm{E}_{\mathrm{i}} \rightarrow \mathrm{E}_{\mathrm{i}} /\left(1-v_{\mathrm{i}}{ }^{2}\right)$ and $\mathrm{d}_{31} \rightarrow \mathrm{d}_{31}\left(1+v_{\mathrm{i}}\right)$, where $v_{\mathrm{i}}$ is the material Poisson's ratio. For our doubly-fixed membrane design parameters $\left(\mathrm{d}_{31}=-100 \mathrm{pC} / \mathrm{N}, \mathrm{E}_{\mathrm{pzt}}=90 \mathrm{GPa}, \mathrm{t}_{\mathrm{pzt}}\right.$ $=0.5 \mu \mathrm{m}$, and $\mathrm{L}=450 \mu \mathrm{m})$, our analytical and finite-element Coventorware models are in agreement to within $0.5 \%$. Both predict a $250 \mathrm{~nm}$ x-axis displacement of the membrane with a single actuator at $10 \mathrm{~V}$ applied and suggest a $1.83 \mathrm{~nm}$ grating period change $(0.046 \%$ strain), assuming strain uniformity across the membrane. For a $632.8 \mathrm{~nm}$ laser on a 4 um grating period, this corresponds to an angular change of $72 \mu$ radian for the first diffracted order.

Moreover, the finite-element model, calibrated against our experimental results, provides a numerical estimate on the slight bow in the membrane, at $0.94 \mu \mathrm{m}$ raise against the edges of a 232 $\mu \mathrm{m}$ bow length. Approaching the diffracted image as the Fourier transform of the binary phase grating in the Fraunhofer regime [7], we estimate a decrease in first order diffraction efficiency to about $4.4 \%$ when actuated at $10 \mathrm{~V}$. In terms of mechanical resolution, however, the fine voltage control of the piezoelectric material suggests the resolution of a grating period change below a nanometer and angular change below a microradian. Out-of-plane membrane displacement, asymmetrical rotation of the membrane when actuated, thermal disturbances, modal vibrations, and optical detection techniques will limit the finest achievable resolution of the total membrane displacement.

\section{FABRICATION}

The fabrication process for the electrostatic device is depicted in Figure 3. It starts with an SOI (Silicon On Insulator) wafer with a $20 \mu \mathrm{m}$ thick device layer and a $2 \mu \mathrm{m}$ thick buried oxide. First we etch through the device layer with DRIE (Deep Reactive Ion Etching) technology. The advantage of using DRIE is that it allows us to obtain grating beams and flexures that are $20 \mu \mathrm{m}$ thick in the vertical direction. This increases the vertical stiffness of the structure and avoids potential stiction problems during the releasing step. The design also includes lateral bumps to ensure that no lateral stiction occurs either. More importantly, the device is essentially free of residual stress because the main structure, i.e., the device silicon layer, is not formed by thin-film deposition. Since the buried oxide behaves essentially like a good etch stop, our design also avoids potential problems due to the loading effect (i.e., etching non-uniformity due to different exposed areas) which shows up almost in any etching process. The DRIE process is followed by an HF etching step to release the moving parts. Since the lateral dimension of the moving parts is much smaller than that of the fixed parts, we have large process latitude during the timecontrol releasing process. After releasing, we deposit an aluminum film to form the electrodes and the reflective surface on the gratings. Figures 4 shows images of the fabricated electrostatic device.

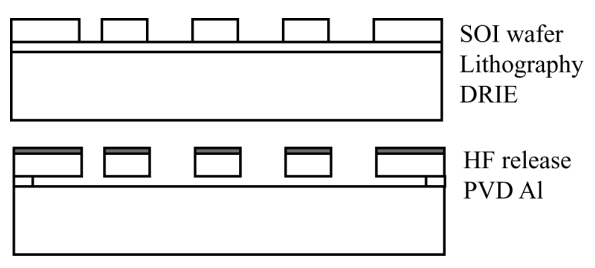

Figure 3. Electrostatic device fabrication process flow. (a)

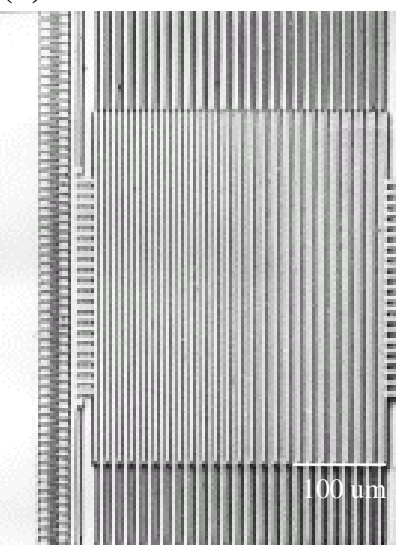

(b)

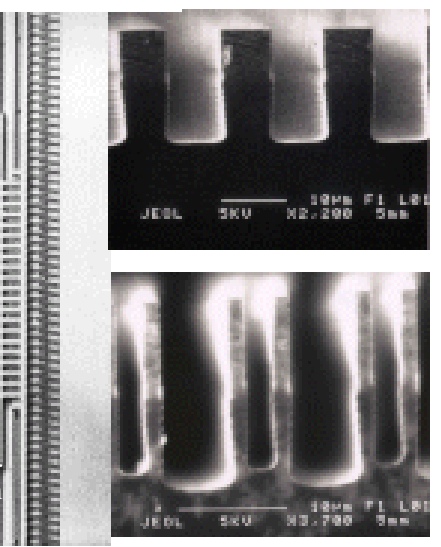

Figure 4. Tunable electrostatic grating: (a) under optical microscope at 130X magnification, (b) SEM cross-sections of the gratings (top) and the flexures (bottom) on test sample.

Fabrication process of the piezoelectric analog tunable grating, shown in Figure 5, involves both surface and bulk micromachining to improve device yield. First, a $200 \mathrm{~nm}$ silicon nitride layer is deposited via PECVD and then patterned to form a hard-mask for a later $\mathrm{KOH}$ backside etch. A $220 \mathrm{~nm} \mathrm{Pt/Ti} \mathrm{layer} \mathrm{is}$ then evaporated on the substrate and patterned to create the bottom electrode via lift-off, instead of RIE, to facilitate fabrication process repeatability. Sol-gel PZT is subsequently spun-on and annealed in repeated individual steps to create a high-quality PZT layer with $0.5 \mu \mathrm{m}$ thickness. PZT hillock and crack formation issues were resolved with fine-tuning of the Pt/Ti bottom electrode and PZT fabrication conditions. The piezoelectric layer is successfully patterned via an economic wet-etch technique [8] and the top electrode deposited with a second $220 \mathrm{~nm}$ Pt/Ti evaporation and lift-off procedure. The diffractive grating is next separately created with a $160 \mathrm{~nm}$ Pt lift-off with $2 \mu \mathrm{m}$ minimal linewidth features. The doubly-hinged membrane is then defined with a 445 um $\mathrm{KOH}$ backside etch, and released with a $5 \mu \mathrm{m}$ Si RIE.

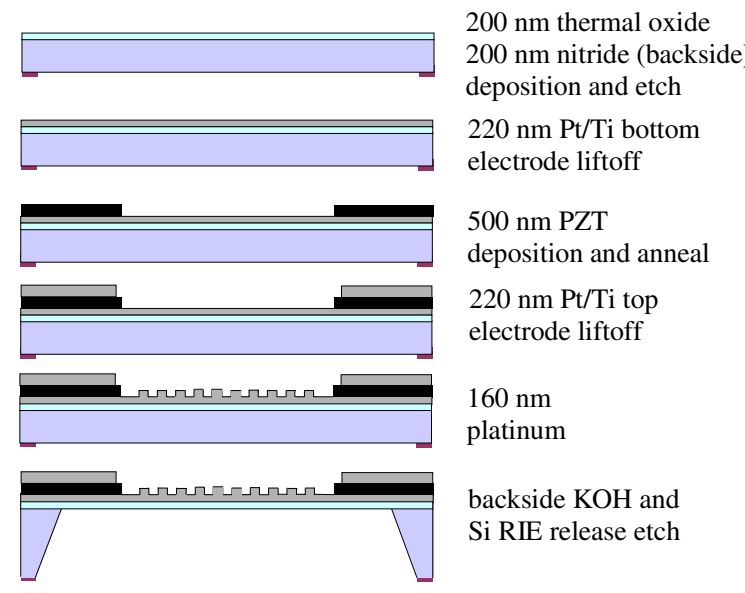

Figure 5. Piezoelectric device fabrication process flow. 
The fabricated device is shown in Figure 6. The completed PZT has a predominant perovskite phase aided by good adhesion between the bottom $\mathrm{Pt} / \mathrm{Ti}$ electrode, the $\mathrm{SiO}_{2}$ diffusion barrier, and the substrate. The average grain size of the PZT film is on the order of $0.1 \mu \mathrm{m}$. Ferroelectric characterization, Figure 7 , suggests a excellent dielectric constant of above 1200 and a dielectric loss below 0.05. Fatigue lifecycle experiments suggest operation above $10^{10}$ cycles under a $5 \mathrm{~V}$ rectangular pulse-train signal. The power consumption of the PZT film at $10 \mathrm{~V}$ actuation is gauged at $30 \mathrm{nW}$, made possible from its high resistivity. The fabricated binary phase grating also has a duty cycle measured as varying between 42 to $66 \%$, depending on the process conditions. First and second order diffraction efficiencies were measured at $7.6 \%$ and $5.0 \%$ respectively for the fabricated grating, although higher efficiencies could be achieved with better process control.

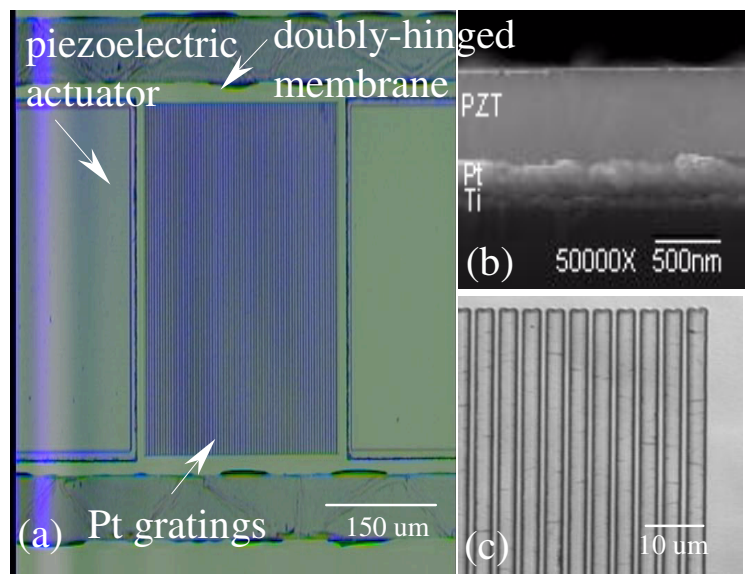

Figure 6. (a) Piezoelectric-actuated tunable grating under $120 X$ magnification, (b) SEM cross-section image of fabricated PZT actuator on Pt/Ti electrodes, (c) magnified view of Pt gratings with 4 um period.

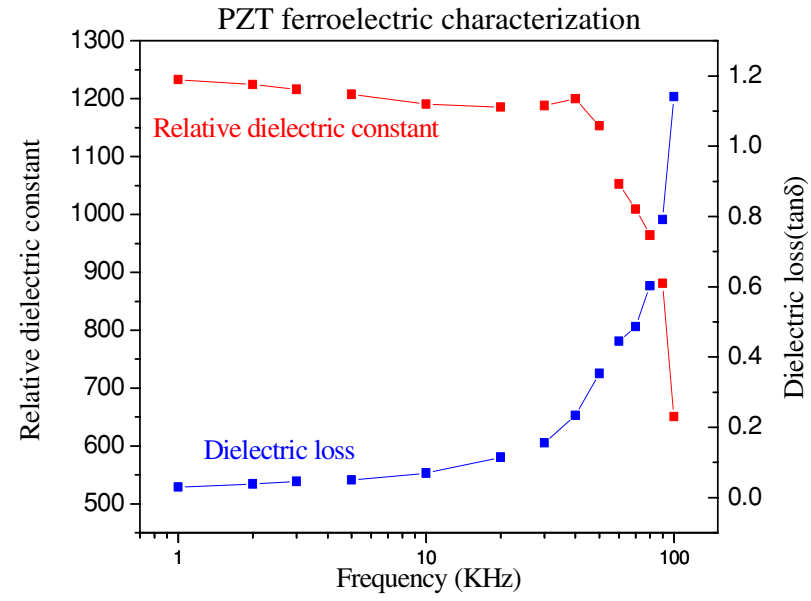

Figure 7. Ferroelectric response of fabricated PZT film. A relative dielectric constant of 1200 and dielectric loss of less than 0.05 was measured.

\section{EXPERIMENTAL DESCRIPTION}

We measured the angle change versus actuation voltage with two different methods: optical beam steering with image processing, and the computer microvision technique [9], which involves obtaining three-dimensional images of microscopic targets using the optical sectioning property of a light microscope and post-processing the combined images to analyze the images with nanometer precision.

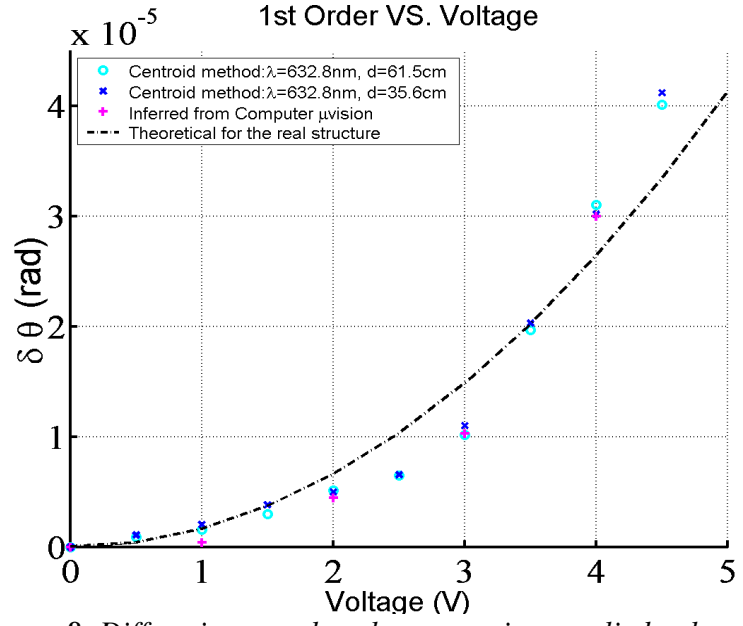

Figure 8. Diffraction angular change against applied voltage for electrostatic device. The centroid method compares the first-order image centroids before and after actuation.

The results of the electrostatic device (deflection angle vs. voltage) from two testing setups, Figure 8 , match the theory very well. The tuning range is about $0.05 \mathrm{mrad}$ at $5 \mathrm{~V}$ and the resolution is roughly an order of magnitude smaller. The measured frequency response, Figure 9, has the first mode at $1-2 \mathrm{KHz}$ due to the very compliant flexure. Figure 10 shows the measured diffraction efficiency over different actuation voltages. Both device design and fabrication process optimizations are underway.

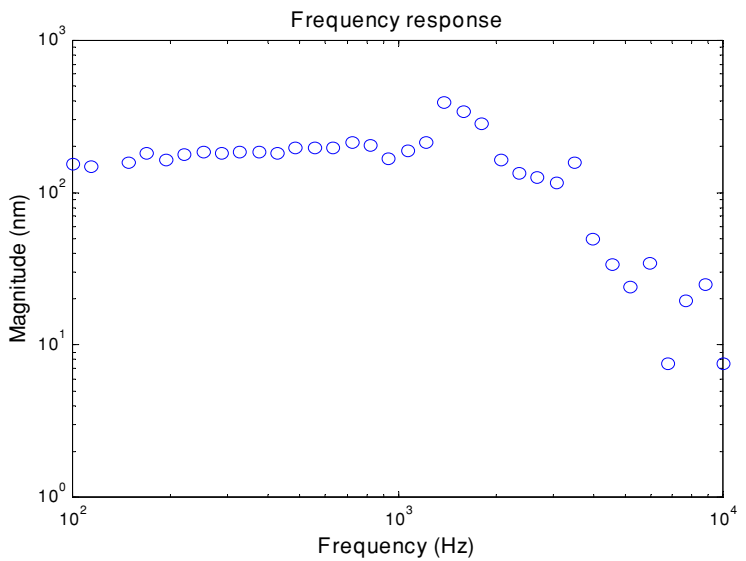

Figure 9. Frequency response of electrostatic gratings, depicting resonance above operating range. This device has 7 periods.

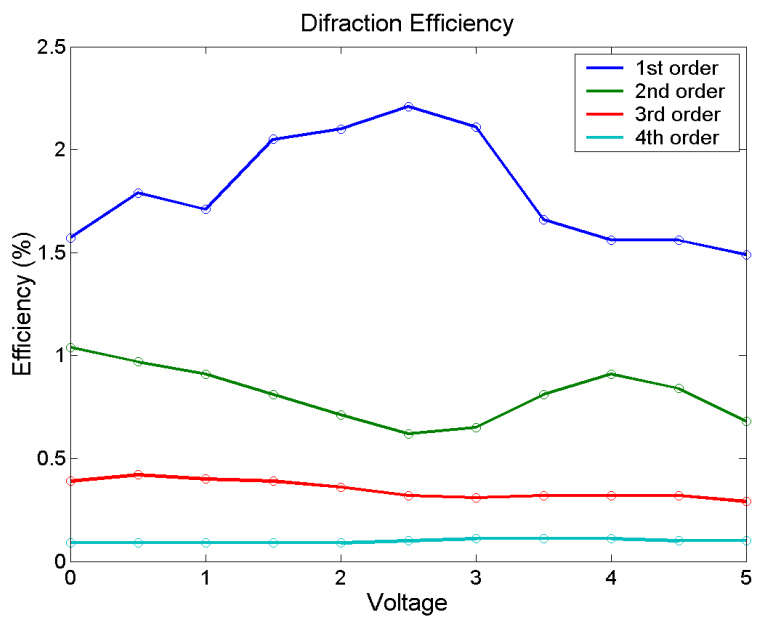

Figure 10. Diffraction efficiency of electrostatic grating against applied voltage. The efficiency is lower than previously measured values of $12 \%$ due to the absence of the reflective metal deposition in this device batch. 
The membrane deformation of the piezoelectric-actuated device, measured with the computer microvision technique [9], demonstrated a $229 \pm 2 \mathrm{~nm}$ in-plane motion along the membrane length at $9.0 \mathrm{~V}$ actuation. This corresponds to an estimated period change of $1.8 \mathrm{~nm}$ for this set of devices, assuming uniformity in the membrane strain. Measurements on uniformity suggest a variation of approximately $10 \%$ across the gratings. The gratings also show an expected shrinkage in the y-lateral direction due to deformation in the x-lateral direction. The membrane deformation is in agreement with the analytical and finite-element formulation and a more detailed characterization of the $\mathrm{d}_{31}$ coefficient will be carried out. In Figure 11, the PZT polarization-electric field hysteresis behavior, due to the domain reorientations, is evident when the membrane is actuated from $-10 \mathrm{~V}$ to $+10 \mathrm{~V}$. This matches with the coercive field from electrical hysteresis

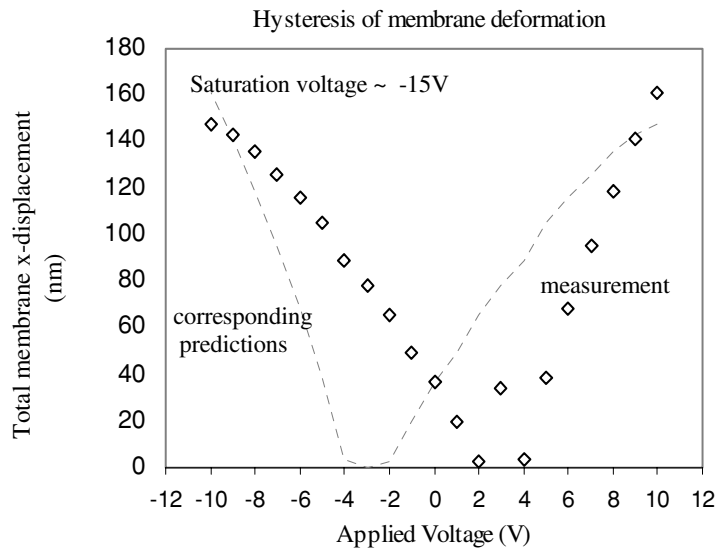

Figure 11. Deformation pulled with saturation voltage $(-15 \mathrm{~V})$ and step-wise increased to $10 \mathrm{~V}$ (measured with computer microvision [9]). The non-zero displacement at $0 \mathrm{~V}$ results from hysteresis in the PZT.

In addition, the diffraction angles agree well with diffraction theory. More specifically, the angular change of the first diffracted order by applying voltage to one of the two actuators in the design is shown in Figure 12. Comparisons between the optical image centroid processing, mechanical membrane deformation measurements, and theoretical predictions suggest good agreement. At $10 \mathrm{~V}$ actuation, the angular change is estimated at $75 \mu$ radians for this set of devices. The uncertainty is largely limited to tilt in the membrane when actuated. Future work involves obtaining detailed measurements of the out-of-plane motion and bow of the actuated membrane.

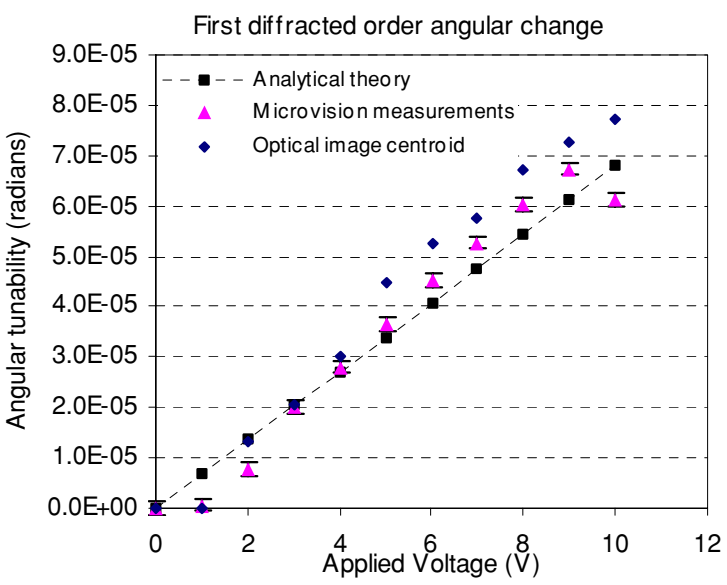

Figure 12. First order diffracted angular change against applied voltage obtained by optical image centroid processing and mechanical motion measurements. The results are in good

\section{CONCLUSIONS}

We have designed, fabricated, and demonstrated electrostatic and piezoelectric-actuated analog tunable gratings, via mechanical deformation of the grating structure. The expected performance of the electrostatic-actuated device is a wide tunable range of milliradians with resolution of three orders of magnitude smaller at actuation voltage below $30 \mathrm{~V}$ with closed-loop control. The piezoelectric-actuated tunable grating, moreover, demonstrates a grating period change on the order of a nanometer, agreeing well with our analytical and finite-element models, and suggests a range up to 0.1 milliradian with resolution on the order of microradians.

\section{ACKNOWLEDGEMENTS}

The authors would like to thank Arnab Sinha, Kurt Broderick and Martin Schmidt for their invaluable assistance. The contributions by Gregory Nielson and Carlos Hidrovo are also appreciated. This project is funded by ASML and the National Science Foundation.

\section{REFERENCES}

1. $\quad$ R. B. Apte et al., "Grating Light Valves for High Resolution Displays," Solid State Sensors and Actuators Workshop, Hilton Head, SC, pp.1-6, June 1994.

2. M. B. Sinclair et al., "Synthetic Spectra: A Tool for Correlation Spectroscopy," Applied Optics 36, No. 15, pp.3342-3348, May 1997.

3. X.M. Zhang and A.Q. Liu, "A MEMS Pitch-tunable Grating Add / Drop Multiplexers", Optical MEMS 2000 IEEE/LEOS International Conference, Kauai, HI, pp.25-26, August 2000.

4. D.E. Sene et al., "Development and Characterization of Micro-Mechanical Gratings for Optical Modulation", Proc. of IEEE MEMS Workshop, San Diego, CA, pp.222-227, February 1996.

5. W. C. Tang et al., "Lateral Driven Polysilicon Resonant Microstructures," Proc. of IEEE MEMS Workshop, Salt Lake City, UT, pp.53-59, February 1989.

6. M. S. Weinberg, "Working Equations for Piezoelectric Actuators and Sensors", Jour. of Microelectromechanical Systems, Vol.8, No.4, pp.529-533, December 1999.

7. J.W. Goodman, Introduction to Fourier Optics, McGraw-Hill, San Francisco, CA, pp.63-89, $2^{\text {nd }}$ edition, 1996.

8. W. Liu et al., "Preparation and properties of multiplayer $\mathrm{Pb}(\mathrm{Zr}, \mathrm{Ti}) \mathrm{O} 3$ / $\mathrm{PbTiO} 3$ thin films for pyroelectric application", Thin Solid Films, V371, Issue 1-2, pp.254-258, August 2000.

9. D. M. Freeman et al., "Multidimensional Motion Analysis of MEMS using Computer Microvision," Solid State Sensors and Actuators Workshop, Hilton Head, SC, pp.150-155, June 1998. 